\title{
Conformal wave expansions for flat space amplitudes
}

\author{
Chang Liu and David A. Lowe \\ Department of Physics, Brown University, \\ Providence, RI, 02912, U.S.A. \\ E-mail: chang_liu3@brown.edu, lowe@brown.edu
}

ABSTRACT: The extended BMS algebra contains a conformal subgroup that acts on the celestial sphere as $\mathrm{SO}(3,1)$. It is of interest to perform mode expansions of free fields in Minkowski spacetime that realize this symmetry in a simple way. In the present work we perform such a mode expansion for massive scalar fields using the unitary principal series representations of $\mathrm{SO}(3,1)$ with a view to developing a holographic approach to gravity in asymptotically flat spacetime. These mode expansions are also of use in studying holography in three-dimensional de Sitter spacetime.

KEYWORDS: Gauge-gravity correspondence, Classical Theories of Gravity

ArXiv ePrint: 2105.01026 


\section{Contents}

1 Introduction 1

2 Conformal coordinates $\quad 2$

3 3d de Sitter mode functions $\quad 4$

4 Uplifting onto 4d Minkowski $\quad 5$

5 Klein-Gordon norm and orthonormality conditions $\quad 6$

$6 \quad$ Unitary principal series representation $\quad 9$

7 Relation to previous work 12

8 Discussion $\quad \mathbf{1 4}$

8.1 Holographic mapping between 3D de Sitter spacetime and a Euclidean 2-sphere 14

8.2 Holographic mapping between celestial sphere and 4D Minkowski spacetime 15

A Euclidean vacuum

\section{Introduction}

There has been considerable interest recently in constructing holographic theories between flat 4d Minkowski spacetime and a $2 \mathrm{~d}$ boundary celestial sphere conformal field theory [14]. Central to this mission is the construction of conformally covariant wavefunctions that form unitary representations of the $\mathrm{SO}(d, 1)$ group. These wavefunctions are defined on a $d$-dimensional de Sitter spacetime $\mathrm{dS}_{d}$, on which $\mathrm{SO}(d, 1)$ acts naturally through an embedding of $\mathrm{dS}_{d}$ as a submanifold of a $(d+1)$-dimensional Minkowski spacetime.

As part of the program to realize the dS/CFT correspondence [5], numerous papers have previously constructed unitary principal series representations of the $\mathrm{SO}(2,1)$ group on two-dimensional de Sitter spacetimes [6, 7], as well as $q$-deformed versions of the principal series on the three-dimensional de Sitter spacetime [8]. In this paper we construct the unitary principal series representation of the $\mathrm{SO}(3,1)$ group on the three-dimensional de Sitter spacetime. We also compute the uplifted version of these wavefunctions on the ambient four-dimensional Minkowski spacetime. Finally, we comment on relevant previous work [9-11] and discuss how our results fit within the program to develop holographic approaches to gravity in de Sitter and asymptotically Minkowski spacetime.

The sections are organized as follows: we first establish our coordinate systems and fix our notations in section 2 . We then construct massive scalar mode functions on both the 
three-dimensional de Sitter spacetime $\mathrm{dS}_{3}$ and the four-dimensional Minkowski spacetime $\mathrm{M}_{4}$, in section 3 and 4 , respectively. We then show in section 6 that these mode functions form a unitary principal series representation of the $\mathrm{SO}(3,1)$ group. We note that previous work [9-11] uses modes that form non-unitary highest weight representations of $\mathrm{SO}(3,1)$. Finally we comment in section 8 on how our mode functions can serve as a conformal basis to develop holographic formulations of gravity in asymptotically de Sitter and Minkowski spacetimes.

\section{Conformal coordinates}

Before we begin our discussion of the representation theory of $\mathrm{SO}(3,1)$ in de Sitter and Minkowski spacetimes, we would like to present the coordinate systems we employ and fix notation. A schematic plot of the relevant hypersurfaces can be found in figure 1 .

We start with the $4 \mathrm{~d}$ flat Minkowski spacetime labeled by coordinates $\left(x^{0}, x^{1}, x^{2}, x^{3}\right)$ with the following metric

$$
\mathrm{d} s^{2}=-\left(\mathrm{d} x^{0}\right)^{2}+\left(\mathrm{d} x^{1}\right)^{2}+\left(\mathrm{d} x^{2}\right)^{2}+\left(\mathrm{d} x^{3}\right)^{2} .
$$

We embed our 3d de Sitter spacetime as a hypersurface within the $4 \mathrm{~d}$ Minkowski spacetime. To do so we first switch to hyperbolic coordinates $(t, \rho, \theta, \varphi)$ with $-\infty<t<\infty, \rho>0$, $0 \leq \theta<\pi$ and $0 \leq \varphi<2 \pi$, defined by

$$
\begin{aligned}
& x^{0}=\rho \sinh (t / \rho) \\
& x^{1}=\rho \cos \theta \cosh (t / \rho) \\
& x^{2}=\rho \sin \theta \cos \varphi \cosh (t / \rho) \\
& x^{3}=\rho \sin \theta \sin \varphi \cosh (t / \rho) .
\end{aligned}
$$

Note that these coordinates only cover the region of the Minkowski spacetime defined by points $x^{\mu}$ where $x \cdot x>0$. The metric then becomes

$$
\mathrm{d} s^{2}=-\mathrm{d} t^{2}+\frac{2 t \mathrm{~d} \rho \mathrm{d} t}{\rho}+\left(1-\frac{t^{2}}{\rho^{2}}\right) \mathrm{d} \rho^{2}+\rho^{2} \cosh ^{2}\left(\frac{t}{\rho}\right)\left(\mathrm{d} \theta^{2}+\sin ^{2} \theta \mathrm{d} \varphi^{2}\right) .
$$

The d'Alembertian in this coordinate system looks rather complicated. We therefore perform the following change of variables

$$
\eta=\frac{t}{\rho}
$$

and the metric takes the following simpler form

$$
\mathrm{d} s^{2}=-\rho^{2} \mathrm{~d} \eta^{2}+\mathrm{d} \rho^{2}+\rho^{2} \cosh ^{2} \eta\left(\mathrm{d} \theta^{2}+\sin ^{2} \theta \mathrm{d} \varphi^{2}\right) .
$$

Note a constant $\eta$ surface forms a Cauchy slice. This will be important in the following, so that free fields states on such spacelike slices provide a complete set. One might instead choose Euclidean hyperbolic spacelike slices in the upper or lower triangles of figure 1. This 

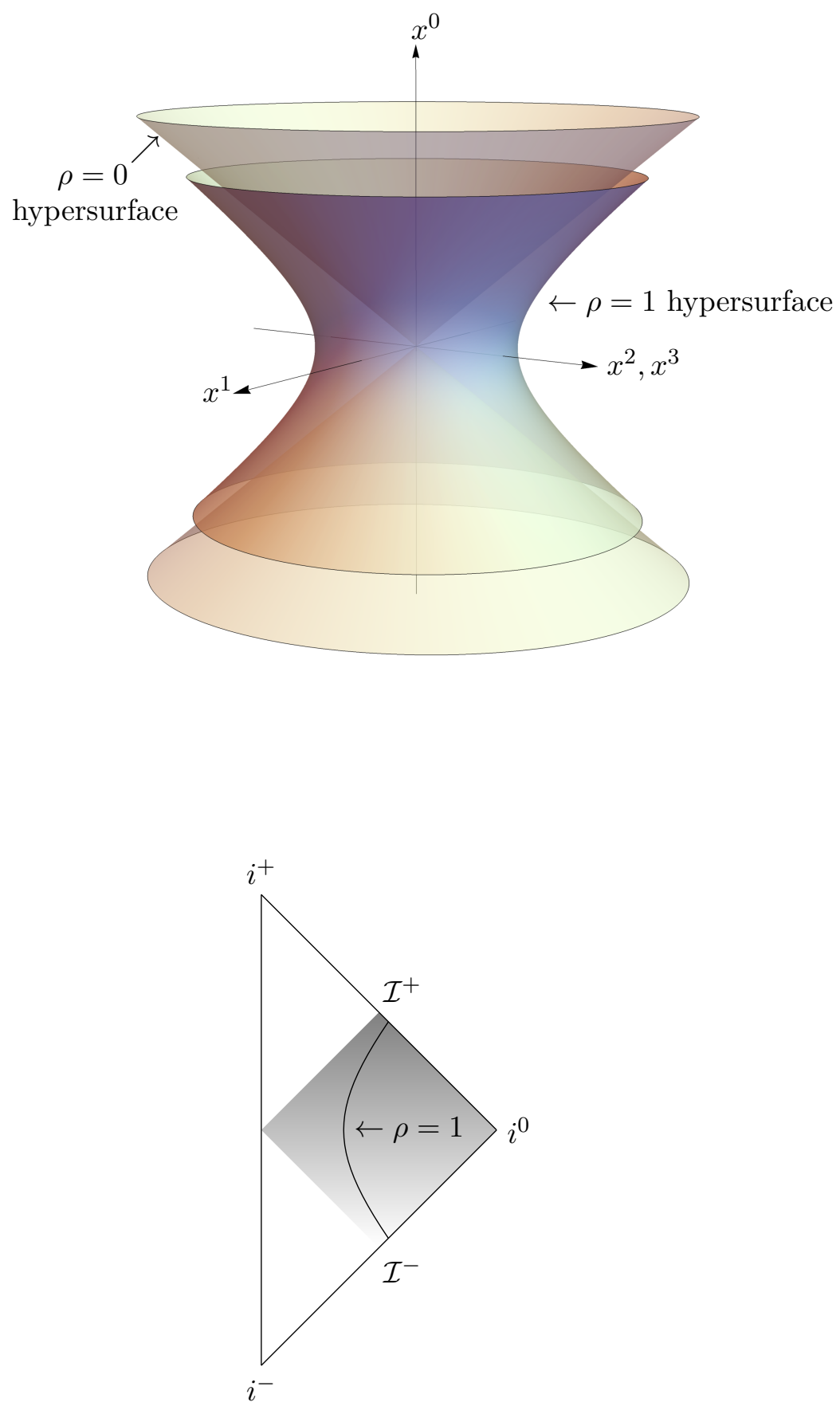

Figure 1. Minkowski spacetime may be divided up into radial $(\rho)$ slices isometric to 3d de Sitter spacetime as shown in the top panel. The bottom panel shows the Penrose diagram of Minkowski spacetime. The shaded region is the region bounded by $\rho=0$ and $\rho=\infty$. In particular, $i^{ \pm}$are excluded from this region. 
is the case primarily studied in [1]. Such slices transform in a simple way under $\mathrm{SO}(3,1)$, but do not form Cauchy slices and will not lead to a complete set of free field states.

A $3 \mathrm{~d}$ de Sitter spacetime can then be embedded into this 4d Minkowski spacetime as a hypersurface of constant $\rho=\ell$, where $\ell$ is the de Sitter length scale which we will set to unity in what follows. On the $3 \mathrm{~d}$ de Sitter spacetime the induced metric is simply

$$
\mathrm{d} s^{2}=-\mathrm{d} t^{2}+\cosh ^{2} t\left(\mathrm{~d} \theta^{2}+\sin ^{2} \theta \mathrm{d} \varphi^{2}\right) .
$$

Through a stereographic projection we can parameterize the 2 -sphere covered by coordinates $(\theta, \varphi)$ with a complex variable $z$, and obtain the Fubini-Study metric on the 2 -sphere:

$$
\mathrm{d} \theta^{2}+\sin ^{2} \theta \mathrm{d} \varphi^{2}=\frac{4 \mathrm{~d} z \mathrm{~d} \bar{z}}{\left(1+|z|^{2}\right)^{2}} .
$$

This allows us to rewrite the $3 \mathrm{~d}$ de Sitter metric as

$$
\mathrm{d} s^{2}=-\mathrm{d} t^{2}+\cosh ^{2} t \frac{4 \mathrm{~d} z \mathrm{~d} \bar{z}}{\left(1+|z|^{2}\right)^{2}}
$$

and the $4 \mathrm{~d}$ Minkowski metric in hyperbolic coordinates as

$$
\mathrm{d} s^{2}=-\rho^{2} \mathrm{~d} \eta^{2}+\mathrm{d} \rho^{2}+\rho^{2} \cosh ^{2} \eta \frac{4 \mathrm{~d} z \mathrm{~d} \bar{z}}{\left(1+|z|^{2}\right)^{2}} .
$$

\section{3d de Sitter mode functions}

We start from the metric on the $3 \mathrm{~d}$ de Sitter spacetime (2.2). The isometry group of the $3 \mathrm{~d}$ de Sitter spacetime is $\mathrm{SO}(3,1)$ which has 6 generators with real coefficients. The first step in building a unitary representation of $\mathrm{SO}(3,1)$ on $3 \mathrm{~d}$ de Sitter is to solve the scalar field equation with mass $\mu$

$$
\left(\Delta-\mu^{2}\right) \phi(t, z, \bar{z})=0
$$

where the d'Alembertian is defined in general as

$$
\Delta \phi=\frac{1}{\sqrt{|g|}} \partial_{i}\left(g^{i j} \sqrt{|g|} \partial_{j} \phi\right) .
$$

Here and in what follows we use Latin indices when we are referring to the 3d de Sitter submanifold and use Greek indices when we are dealing with the ambient 4d Minkowski spacetime. The d'Alembertian is computed to be

$$
\Delta \phi=\left[-\partial_{t}^{2}-2(\tanh t) \partial_{t}+\left(\operatorname{sech}^{2} t\right)\left(1+|z|^{2}\right)^{2} \partial_{z} \partial_{\bar{z}}\right] \phi .
$$

The massive scalar field equation therefore is

$$
\left(-\frac{\partial^{2}}{\partial t^{2}}-2 \tanh t \frac{\partial}{\partial t}+\operatorname{sech}^{2} t\left(1+|z|^{2}\right)^{2} \frac{\partial^{2}}{\partial z \partial \bar{z}}-\mu^{2}\right) \phi(t, z, \bar{z})=0 .
$$

We can perform separation of variables as usual and write a mode function as

$$
\phi_{l m}(t, z, \bar{z})=\phi_{l}(t) Y_{m}^{l}(z, \bar{z})
$$


where $m$ is a $j_{3}$ eigenvalue. The $Y_{m}^{l}$ are the standard spherical harmonics [12] in $(z, \bar{z})$ coordinates. They satisfy the eigenvalue equation

$$
\left(1+|z|^{2}\right)^{2} \frac{\partial^{2}}{\partial z \partial \bar{z}} Y_{m}^{l}=-l(l+1) Y_{m}^{l}
$$

Explicitly, we have

$$
Y_{m}^{l}(\theta, \varphi)=\sqrt{\frac{(2 l+1)(l-m) !}{4 \pi(l+m) !}} P_{l}^{m}(\cos \theta) e^{i m \varphi}
$$

where

$$
P_{l}^{m}(x)=\frac{(1+x)^{m / 2}}{(1-x)^{m / 2}} \sum_{k=0}^{l} \frac{\left(\frac{1-x}{2}\right)^{k}(-l)_{k}(l+1)_{k}}{k ! \Gamma(k-m+1)} .
$$

The coordinate transformation linking $(\theta, \varphi)$ and $(z, \bar{z})$ is

$$
\left(\sin \theta e^{i \varphi}, \cos \theta\right)=\left(\frac{2 z}{1+|z|^{2}}, \frac{1-|z|^{2}}{1+|z|^{2}}\right) .
$$

It is easy to see that the spherical harmonics $Y_{l}^{m}(z, \bar{z})$ can be built out of homogeneous polynomials of the following three variables

$$
F_{z}=\frac{2 z}{1+|z|^{2}} \quad F_{\bar{z}}=\frac{2 \bar{z}}{1+|z|^{2}} \quad F_{t}=\frac{1-|z|^{2}}{1+|z|^{2}} .
$$

We will later see that these three variables will appear in (6.1). One is then left to solve

$$
\left(\frac{\partial^{2}}{\partial t^{2}}+2 \tanh t \frac{\partial}{\partial t}+l(l+1) \operatorname{sech}^{2} t+\mu^{2}\right) \phi_{l}(t)=0 .
$$

This has the following two linearly independent solutions

$$
\phi_{l, 1}(t)=\operatorname{sech} t P_{l}^{\sqrt{1-\mu^{2}}}(\tanh t), \quad \phi_{l, 2}(t)=\operatorname{sech} t Q_{l}^{\sqrt{1-\mu^{2}}}(\tanh t)
$$

where $P$ and $Q$ are the associated Legendre functions of first and second kind, respectively. We expect to get a unitary representation corresponding to a general complex linear combination, one of which will be the Euclidean vacuum (see section 5 and appendix A). Other combinations will generate modes around an $\alpha$-vacuum [13-15].

\section{Uplifting onto 4d Minkowski}

To uplift the $3 \mathrm{~d}$ de Sitter mode functions onto the 4d Minkowski spacetime we consider the scalar field equation in $4 \mathrm{~d}$ with mass $M$

$$
\left(\square-M^{2}\right) \Phi(\eta, \rho, z, \bar{z})=0 .
$$

The d'Alembertian is computed to be

$$
\square=-\frac{1}{\rho^{2}}\left(\partial_{\eta}^{2}+2 \tanh \eta \partial_{\eta}\right)+3 \frac{\partial_{\rho}}{\rho}+\partial_{\rho}^{2}+\frac{\operatorname{sech}^{2} \eta\left(1+|z|^{2}\right)^{2} \partial_{z} \partial_{\bar{z}}}{\rho^{2}} .
$$


Separating variables, the mode functions can be written as

$$
\Phi_{p l m}(\eta, \rho, z, \bar{z})=\phi_{p l}(\eta) \psi_{p}(\rho) Y_{m}^{l}(z, \bar{z})
$$

where $l=0,1, \cdots, m=-l, \cdots, l$ and the range of the real parameter $p$ will be discussed later. We find the differential equation for $\phi_{p l}$ to be identical to the $3 \mathrm{~d}$ de Sitter modes, except that we replace $\mu$ with a real parameter $p$,

$$
\left(\partial_{\eta}^{2}+2(\tanh \eta) \partial_{\eta}+l(l+1) \operatorname{sech}^{2} \eta+p^{2}\right) \phi_{p l}(\eta)=0
$$

which has solutions (3.1) with the replacement $t \rightarrow \eta$ and $\mu \rightarrow p$.

The differential equation for $\psi_{p}$ therefore becomes

$$
\left(\partial_{\rho}^{2}+\frac{3}{\rho} \partial_{\rho}+\frac{p^{2}}{\rho^{2}}-M^{2}\right) \psi_{p}(\rho)=0
$$

This has two independent solutions

$$
\psi_{p, 1}=\frac{I \sqrt{1-p^{2}}(M \rho)}{\rho}, \quad \psi_{p, 2}=\frac{K \sqrt{1-p^{2}}(M \rho)}{\rho}
$$

where $I_{\alpha}$ and $K_{\alpha}$ are modified Bessel functions of first and second kind, respectively.

\section{Klein-Gordon norm and orthonormality conditions}

Before presenting the explicit form of our unitary principal series representation we would like to first establish the orthonormalizability of the $4 \mathrm{~d}$ Minkowski mode functions. The mode functions are normalized with respect to the Klein-Gordon norm, the most general form of which is [16]

$$
\langle f, g\rangle=-i \int_{\Sigma} \mathrm{d} \Sigma n^{\lambda}\left(f \partial_{\lambda} g^{\star}-g^{\star} \partial_{\lambda} f\right) .
$$

Here $\Sigma$ is a spacelike surface, $n^{\lambda}$ is a timelike unit vector field normal to $\Sigma$ and $d \Sigma$ is the volume element in $\Sigma$. This norm is time-independent, and in principle can be evaluated on any Cauchy slice. Here, for convenience we choose the $\eta=\eta_{0}$ slice, where $\eta_{0}$ is an arbitrary constant. On the $4 \mathrm{~d}$ Minkowski spacetime with coordinate system $(\eta, \rho, \theta, \varphi)$ this then becomes

$$
\langle f, g\rangle=-\left.i \int_{\rho} \int_{S^{2}}\left(f \partial_{\eta} g^{\star}-g^{\star} \partial_{\eta} f\right)\right|_{\eta=\eta_{0}}\left(\cosh ^{2} \eta_{0}\right) \rho \mathrm{d} \rho \mathrm{d} \Omega
$$

where $\mathrm{d} \Omega$ is the area element of the unit 2 -sphere $S^{2}$. Given that our mode functions are separable $\Phi_{p l m}(\eta, \rho, z, \bar{z})=\phi_{p l}(\eta) \psi_{p}(\rho) Y_{m}^{l}(z, \bar{z})$ we can further evaluate this norm to obtain

$$
\begin{aligned}
\left\langle\Phi_{p l m}, \Phi_{p^{\prime} l^{\prime} m^{\prime}}\right\rangle= & -i\left(\phi_{p l}\left(\eta_{0}\right) \dot{\phi}_{p^{\prime} l^{\prime}}^{\star}\left(\eta_{0}\right)-\phi_{p^{\prime} l^{\prime}}^{\star}\left(\eta_{0}\right) \dot{\phi}_{p l}\left(\eta_{0}\right)\right)\left(\cosh ^{2} \eta_{0}\right) \\
& \times \int_{0}^{\infty} \psi_{p}(\rho) \psi_{p^{\prime}}^{\star}(\rho) \rho \mathrm{d} \rho \int_{S^{2}} Y_{m}^{l}(z, \bar{z}) Y_{m^{\prime}}^{\star l^{\prime}}(z, \bar{z}) \mathrm{d} \Omega
\end{aligned}
$$


where $\dot{\phi}(\eta)=\partial_{\eta} \phi$. The orthonormality of the spherical harmonics allows us to conclude that

$$
\int_{S^{2}} Y_{m}^{l}(z, \bar{z}) Y_{m^{\prime}}^{\star l^{\prime}}(z, \bar{z}) \mathrm{d} \Omega=\delta_{l l^{\prime}} \delta_{m m^{\prime}}
$$

For the $\rho$ integral, note that multiplying the differential equation satisfied by $\psi_{p}$ with $\psi_{p^{\prime}}^{\star}$ gives

$$
\psi_{p}^{\prime \prime} \psi_{p^{\prime}}^{\star}+\frac{3}{\rho} \psi_{p}^{\prime} \psi_{p^{\prime}}^{\star}+\left(\frac{p^{2}}{\rho^{2}}-M^{2}\right) \psi_{p} \psi_{p^{\prime}}^{\star}=0
$$

where $\psi^{\prime}=\partial_{\rho} \psi$. Likewise swapping $\psi_{p} \leftrightarrow \psi_{p^{\prime}}^{*}$ and subtracting we have

$$
-\frac{p^{2}-p^{\prime 2}}{\rho^{2}} \psi_{p} \psi_{p^{\prime}}^{\star}=\psi_{p}^{\prime \prime} \psi_{p^{\prime}}^{\star}-\psi_{p^{\prime}}^{\star^{\prime \prime}} \psi_{p}+\frac{3}{\rho} \psi_{p}^{\prime} \psi_{p^{\prime}}^{\star}-\frac{3}{\rho} \psi_{p^{\prime}}^{\star^{\prime}} \psi_{p}
$$

Integrating, we have

$$
-\left(p^{2}-p^{\prime 2}\right) \int_{0}^{\infty} \psi_{p} \psi_{p^{\prime}}^{\star} \rho \mathrm{d} \rho=\int_{0}^{\infty} \mathrm{d} \rho\left[\rho^{3}\left(\psi_{p}^{\prime \prime} \psi_{p^{\prime}}^{\star}-\psi_{p^{\prime}}^{\star^{\prime \prime}} \psi_{p}\right)+3 \rho^{2}\left(\psi_{p}^{\prime} \psi_{p^{\prime}}^{\star}-\psi_{p^{\prime}}^{\star^{\prime}} \psi_{p}\right)\right] .
$$

The integral on the right hand side can be integrated by parts to yield

$$
-\left(p^{2}-p^{\prime 2}\right) \int_{0}^{\infty} \psi_{p} \psi_{p^{\prime}}^{\star} \rho \mathrm{d} \rho=\left.\left[\rho^{3}\left(\psi_{p}^{\prime} \psi_{p^{\prime}}^{\star}-\psi_{p^{\prime}}^{\star^{\prime}} \psi_{p}\right)\right]\right|_{0} ^{\infty} .
$$

The modified Bessel functions have the following mirror symmetry

$$
I_{\alpha}^{\star}(z)=I_{\alpha^{\star}}\left(z^{\star}\right) \quad K_{\alpha}^{\star}(z)=K_{\alpha^{\star}}\left(z^{\star}\right) .
$$

At $z=+\infty, I_{\alpha}(z) \sim e^{z}$ which increases exponentially while $K_{\alpha}(z) \sim e^{-z}$ which decreases exponentially. We therefore discard the $\psi_{p, 1}$ set of solutions as these modes are not normalizable and study the (4.1) solutions $\psi_{p, 2}$. We begin by considering the case $p^{2}>1$ and take the branch $\sqrt{1-p^{2}}=i \sqrt{p^{2}-1}$. Let us define $\alpha=\sqrt{p^{2}-1}$ and henceforth we will drop the 2 subscript on $\psi_{p, 2}$. Near $z=0$, the expansion

$$
K_{\nu}(z)=2^{\nu-1} \Gamma(\nu) z^{-\nu}+2^{-\nu-1} \Gamma(-\nu) z^{\nu}+\cdots
$$

allows us to evaluate the surface term to give

$$
\begin{aligned}
& \lim _{\rho \rightarrow 0} \rho^{3}\left(\psi_{p}^{\prime} \psi_{p^{\prime}}^{*}-\psi_{p^{\prime}}^{*^{\prime}} \psi_{p}\right) \\
& \quad=\lim _{\rho \rightarrow 0} i\left(\alpha+\alpha^{\prime}\right) 2^{-2}\left(\Gamma\left(i \alpha^{\prime}\right) \Gamma(-i \alpha)(M \rho / 2)^{i\left(\alpha-\alpha^{\prime}\right)}-\Gamma(i \alpha) \Gamma\left(-i \alpha^{\prime}\right)(M \rho / 2)^{-i\left(\alpha-\alpha^{\prime}\right)}\right)
\end{aligned}
$$

treating the rapidly oscillating terms in $\alpha$ as vanishing in the sense of a distribution. For $\rho \rightarrow 0$, this is proportional to a sinc representation of the Dirac delta function

$$
\lim _{\rho \rightarrow 0} \rho^{3}\left(\psi_{p}^{\prime} \psi_{p^{\prime}}^{*}-\psi_{p^{\prime}}^{*^{\prime}} \psi_{p}\right)=-\frac{\alpha \Gamma(i \alpha) \Gamma(-i \alpha)}{2} \sin \left[\left(\alpha-\alpha^{\prime}\right) \log (M \rho / 2)\right]
$$

which gives

$$
\int_{0}^{\infty} \psi_{p} \psi_{p^{\prime}}^{\star} \rho \mathrm{d} \rho=\frac{\Gamma(i \alpha) \Gamma(-i \alpha)}{4} \lim _{C \rightarrow \infty} \frac{\sin \left[C\left(\alpha-\alpha^{\prime}\right)\right]}{\alpha-\alpha^{\prime}}
$$


where we have set $C=\log (M \rho / 2)$. Using the following identity

$$
\lim _{C \rightarrow \infty} \frac{\sin (C x)}{x}=\pi \delta(x)
$$

we then have

$$
\int_{0}^{\infty} \psi_{p} \psi_{p^{\prime}}^{\star} \rho \mathrm{d} \rho=\frac{\pi \Gamma(i \alpha) \Gamma(-i \alpha)}{4} \delta\left(\alpha-\alpha^{\prime}\right)=\frac{\pi^{2}}{4 p \sinh \left(\pi \sqrt{p^{2}-1}\right)} \delta\left(p-p^{\prime}\right) .
$$

For $0 \leq p^{2}<1$, instead of oscillating terms in eq. (5.3) we have power-law divergencies, and the mode functions $\psi_{p}$ in this case again are not normalizable.

To conclude, the normalizable radial mode functions arise from $\psi_{p, 2}$ in (4.1) with $p^{2}>1$. The $p$-dependent prefactor in (5.4) may then be absorbed into the normalization of these functions. We assume this has been done, and in the interest of notational clarity we will from now use $\psi_{p}$ to denote the normalized radial mode functions. The normalized radial mode functions in this case will satisfy the following

$$
\int_{0}^{\infty} \psi_{p} \psi_{p^{\prime}}^{\star} \rho \mathrm{d} \rho=\delta\left(p-p^{\prime}\right) .
$$

For the $\eta$ dependence, we form the following linear combination of $\phi_{p l, 1}$ and $\phi_{p l, 2}$ to obtain

$$
\phi_{p l}=\frac{i \pi}{2} \phi_{p l, 1}+\phi_{p l, 2}
$$

which, as we will show in appendix A, are the (unnormalized) modes corresponding to the 4d Minkowski vacuum. Using

$$
\frac{\partial P_{\nu}^{\mu}}{\partial z}=\frac{\nu z P_{\nu}^{\mu}(z)-(\mu+\nu) P_{\nu-1}^{\mu}(z)}{z^{2}-1} \quad \frac{\partial Q_{\nu}^{\mu}}{\partial z}=\frac{\nu z Q_{\nu}^{\mu}(z)-(\mu+\nu) Q_{\nu-1}^{\mu}(z)}{z^{2}-1}
$$

and

$$
P_{\nu}^{\mu}(0)=\frac{\pi^{1 / 2} 2^{\mu}}{\Gamma\left(\frac{1-\mu-\nu}{2}\right) \Gamma\left(\frac{\nu-\mu}{2}+1\right)} \quad Q_{\nu}^{\mu}(0)=-\frac{\pi^{3 / 2} 2^{\mu-1} \tan \frac{\pi(\mu+\nu)}{2}}{\Gamma\left(\frac{1-\mu-\nu}{2}\right) \Gamma\left(\frac{\nu-\mu}{2}+1\right)}
$$

we can evaluate the $\eta$-dependent part of the Klein-Gordon norm to obtain

$$
-i\left[\phi_{p l}\left(\eta_{0}\right) \phi_{p l}^{\star^{\prime}}\left(\eta_{0}\right)-\phi_{p l}^{\star}\left(\eta_{0}\right) \phi_{p l}^{\prime}\left(\eta_{0}\right)\right]\left(\cosh ^{2} \eta_{0}\right)=\pi e^{-\pi \sqrt{p^{2}-1}}
$$

which does not depend on $\eta_{0}$ due to conservation of the Klein-Gordon norm. We can therefore normalize the modes $\phi_{p l}$ by replacing

$$
\phi_{p l} \rightarrow \frac{e^{\frac{\pi}{2} \sqrt{p^{2}-1}}}{\pi^{1 / 2}} \phi_{p l} .
$$

In what follows we will assume that this has been done and in the interest of notational simplicity we will use $\phi_{p l}$ to denote the normalized modes.

To summarize, for $p^{2}>1$ we have constructed mode functions of the $4 \mathrm{~d}$ Klein-Gordon equation corresponding to the Minkowski vacuum, which when restricted to the de Sitter slice $\rho=1$ correspond to the Euclidean vacuum of the $3 \mathrm{~d}$ de Sitter spacetime. These 
modes $\Phi_{p l m}(\eta, \rho, z, \bar{z})=\phi_{p l}(\eta) \psi_{p}(\rho) Y_{m}^{l}(z, \bar{z})$ are normalized with respect to the KleinGordon norm (5.1) with the following orthonormality condition

$$
\left\langle\Phi_{p l m}, \Phi_{p^{\prime} l^{\prime} m^{\prime}}\right\rangle=\delta\left(p-p^{\prime}\right) \delta_{l l^{\prime}} \delta_{m m^{\prime}} .
$$

For $0 \leq p^{2}<1$, the radial mode functions are not normalizable.

\section{$6 \quad$ Unitary principal series representation}

We are now in a position to build the unitary principal series representation of $\mathrm{SO}(3,1)$ which acts on the $\mathrm{dS}_{3} / \mathrm{M}_{4}$ mode functions. Since the action of $\mathrm{SO}(3,1)$ on $\mathrm{M}_{4}$ leaves the radial coordinate $\rho$ invariant, the actions is identical on both $\mathrm{dS}_{3}$ mode functions and on $\mathrm{M}_{4}$ mode functions. For simplicity of presentation we will focus on $\mathrm{dS}_{3}$ modes, but all equations in this section carry over to the $\mathrm{M}_{4}$ modes trivially.

On the past and future null infinities $\mathcal{I}^{ \pm}$of the $3 \mathrm{~d}$ de Sitter spacetime, the Killing vectors can be written as conformal Killing vectors of the spatial 2-sphere

$$
L_{n}=-z^{n+1} \frac{\partial}{\partial z}, \bar{L}_{n}=-\bar{z}^{n+1} \frac{\partial}{\partial \bar{z}}
$$

where $n=0, \pm 1$. However one should use caution in applying this formula. It is correct when acting on the metric, or massless scalars, but as we will see there will be additional terms that must be added depending on the class of functions or fields considered. General complex combinations of these vectors will not preserve the desired reality conditions, so we will need to be careful to construct the correct 6 independent generators that will appear with real coefficients.

To extend these into the bulk of de Sitter it is helpful to arrange them into an $\mathrm{SO}(3)$ corresponding to the isometries of the spatial slices. With the convention $g=\exp (i \theta J)$ we find the generators:

$$
\begin{aligned}
J_{1} & =\frac{i}{2}\left(L_{-1}+L_{1}+\bar{L}_{-1}+\bar{L}_{1}\right) \\
J_{2} & =\frac{1}{2}\left(L_{-1}-L_{1}-\bar{L}_{-1}+\bar{L}_{1}\right) \\
J_{3} & =L_{0}-\bar{L}_{0} .
\end{aligned}
$$

These immediately extend into the bulk without time dependent contributions. This also allows us to read off the conjugation condition to be imposed on the generators. Since the $J_{k}$ are Hermitian we require

$$
L_{n}^{\dagger}=-\bar{L}_{n}, \quad \bar{L}_{n}^{\dagger}=-L_{n} .
$$

The time-dependent Killing vectors take the form

$$
K=F \frac{\partial}{\partial t}+\frac{1}{2}(1+z \bar{z})^{2} \tanh t\left(\partial_{\bar{z}} F\right) \frac{\partial}{\partial z}+\frac{1}{2}(1+z \bar{z})^{2} \tanh t\left(\partial_{z} F\right) \frac{\partial}{\partial \bar{z}}
$$

where $F$ is one of the three solutions

$$
F_{z}=\frac{2 z}{1+|z|^{2}} \quad F_{\bar{z}}=\frac{2 \bar{z}}{1+|z|^{2}} \quad F_{t}=\frac{1-|z|^{2}}{1+|z|^{2}} .
$$


At $\mathcal{I}^{+}$these reduce to

$$
\begin{aligned}
& \tilde{K}_{1}=L_{1}-\bar{L}_{-1} \\
& \tilde{K}_{2}=\bar{L}_{1}-L_{-1} \\
& \tilde{K}_{3}=L_{0}+\bar{L}_{0}
\end{aligned}
$$

when acting on the metric. It is convenient to assemble these into Hermitian linear combinations:

$$
\begin{aligned}
K_{1} & =\frac{1}{2}\left(L_{1}-\bar{L}_{-1}-\bar{L}_{1}+L_{-1}\right) \\
K_{2} & =\frac{i}{2}\left(\bar{L}_{1}-L_{-1}+L_{1}-\bar{L}_{-1}\right) \\
K_{3} & =-i\left(L_{0}+\bar{L}_{0}\right)
\end{aligned}
$$

which at a general spacetime point become

$$
\begin{aligned}
& K_{1}=\frac{1}{2}\left(\frac{2(z-\bar{z})}{1+|z|^{2}} \frac{\partial}{\partial t}-\tanh t\left(\left(z^{2}+1\right) \frac{\partial}{\partial z}-\left(\bar{z}^{2}+1\right) \frac{\partial}{\partial \bar{z}}\right)\right) \\
& K_{2}=\frac{i}{2}\left(\frac{2(z+\bar{z})}{1+|z|^{2}} \frac{\partial}{\partial t}-\tanh t\left(\left(z^{2}-1\right) \frac{\partial}{\partial z}+\left(\bar{z}^{2}-1\right) \frac{\partial}{\partial \bar{z}}\right)\right) \\
& K_{3}=-i\left(\frac{1-|z|^{2}}{1+|z|^{2}} \frac{\partial}{\partial t}-\tanh t\left(z \frac{\partial}{\partial z}+\bar{z} \frac{\partial}{\partial \bar{z}}\right)\right) .
\end{aligned}
$$

Note the generators satisfy the canonical Lorentz algebra

$$
\begin{aligned}
{\left[J_{i}, J_{j}\right] } & =i \epsilon_{i j k} J_{k} \\
{\left[J_{i}, K_{j}\right] } & =i \epsilon_{i j k} K_{k} \\
{\left[K_{i}, K_{j}\right] } & =-i \epsilon_{i j k} J_{k} .
\end{aligned}
$$

On the three-dimensional de Sitter mode functions (3.1) the generators (6.2) take the simplified form

$$
\begin{aligned}
& K_{1}=\frac{1}{2}\left(\frac{2(z-\bar{z})}{1+|z|^{2}}\left(2 h_{+}-2\right)-\left(\left(z^{2}+1\right) \frac{\partial}{\partial z}-\left(\bar{z}^{2}+1\right) \frac{\partial}{\partial \bar{z}}\right)\right) \\
& K_{2}=\frac{i}{2}\left(\frac{2(z+\bar{z})}{1+|z|^{2}}\left(2 h_{+}-2\right)-\left(\left(z^{2}-1\right) \frac{\partial}{\partial z}+\left(\bar{z}^{2}-1\right) \frac{\partial}{\partial \bar{z}}\right)\right) \\
& K_{3}=-i\left(\frac{1-|z|^{2}}{1+|z|^{2}}\left(2 h_{+}-2\right)-\left(z \frac{\partial}{\partial z}+\bar{z} \frac{\partial}{\partial \bar{z}}\right)\right)
\end{aligned}
$$

where we define

$$
2 h_{ \pm}=\frac{d-1}{2} \pm \sqrt{\left(\frac{d-1}{2}\right)^{2}-\mu^{2}}=1 \pm \sqrt{1-\mu^{2}} .
$$

To check this we note that the group of rotations $\mathrm{SO}(3)$ acts straightforwardly in the basis (3.1). The rotation generators also rotate the $K_{i}$ amongst themselves, so we can 
focus on the action of say $K_{3}$ on the mode functions (3.1). It is then straightforward to check that

$$
\frac{\partial}{\partial t} \phi_{l=0}=2\left(h_{+}-1\right) \phi_{l=1}
$$

which holds for the solutions $\phi_{l, 1}$ and $\phi_{l, 2}$ of (3.1) independently and determines the prefactors that appear in (6.3). Likewise, when acting on the four-dimensional Minkowski modes the generators take the exact same expression with $\mu$ replaced by $p$.

To confirm the generators match the principal series we will use the representation of $\mathrm{SO}(3,1)$ on functions $L^{2}\left(S^{2}\right)$. Note this differs from the more common representation on functions $L^{2}(\mathbb{C})$, which would be applicable to the flat slicing of de Sitter. Likewise there is a representation on $L^{2}\left(H^{2}\right)$, though we won't need that here. The upshot of these different realizations of the principal series is that the extra terms in (6.3) take completely different forms.

To realize the representation we consider the cone $C_{+}^{3}$ embedded in $4 \mathrm{~d}$ Minkowski spacetime, where

$$
x_{1}^{2}+x_{2}^{2}+x_{3}^{2}-x_{0}^{2}=0
$$

We then consider the slice through the cone where $x_{0}=1$. This slice is an $S^{2}$ which may be parameterized by coordinates $z$ above using the Fubini-Study metric. The cone maps into itself under $\mathrm{SO}(3,1)$. The principal series may be defined as functions on the slice that behave as [17]

$$
\left(T^{\sigma}(g) f\right)(z)=\alpha(z, g)^{\sigma} f\left(\frac{g^{-1} \cdot z}{\alpha(z, g)}\right)
$$

where $g$ is a $\mathrm{SO}(3,1)$ group element, and $\alpha(z, g)$ is defined to be the rescaling factor needed to return $g^{-1} \cdot x^{\mu}$ to the slice $x_{0}=1$. The action of $\mathrm{SO}(3,1)$ on $z$ is the usual fraction linear transformation, but the factor $\alpha$ depends on which realization of the principal series we are considering. To write the action of $\operatorname{SL}(2, \mathbb{C})$ on the Minkowski coordinates it is helpful to use the familiar representation

$$
x^{\mu}=\frac{1}{2} \operatorname{Tr}\left(M \sigma^{\mu}\right), \quad M=\left(\begin{array}{cc}
x^{0}+x^{3} & x^{1}-i x^{2} \\
x^{1}+i x^{2} & x^{0}-x^{3}
\end{array}\right), \quad \sigma^{\mu}=\left(\mathbb{1}, \sigma^{i}\right)
$$

where $\sigma^{i}$ are the Pauli matrices. Then an $\operatorname{SL}(2, \mathbb{C})$ transformation acts as

$$
M^{\prime}=S M S^{\dagger}
$$

To rescale back to the slice $x^{0}=1$ we rescale to

$$
\tilde{M}^{\prime}=\frac{M^{\prime}}{\frac{1}{2} \operatorname{Tr}\left(M^{\prime}\right)}
$$


Finally, the coordinates on the 2 -sphere $x^{0}=1$ are matched with the Fubini-Study coordinates via

$$
\begin{aligned}
x^{1}+i x^{2} & =\frac{2 z}{1+|z|^{2}} \\
x^{1}-i x^{2} & =\frac{2 \bar{z}}{1+|z|^{2}} \\
x^{3} & =\frac{1-|z|^{2}}{1+|z|^{2}} .
\end{aligned}
$$

From these equations we can read off the factor $\alpha$ and determine the action of the $z$ coordinate. For

$$
S=\left(\begin{array}{ll}
a & b \\
c & d
\end{array}\right),
$$

the fractional linear transformation is

$$
z^{\prime}=\frac{d z+c}{b z+a}
$$

Since we know the group of rotations acts in a straightforward way on the spherical harmonics, it suffice to consider one of the boost generators to check the matching of the generators. To do this we Taylor expand (6.4) for a group element of the form $g=\exp \left(i k_{3} \epsilon\right)$. Plugging in the above relations gives

$$
K_{3}=-i\left(\frac{1-|z|^{2}}{1+|z|^{2}} \sigma-\left(z \frac{\partial}{\partial z}+\bar{z} \frac{\partial}{\partial \bar{z}}\right)\right) .
$$

We therefore identify the scalar field representation with a principal series representation where $\sigma=-2 h_{-}$. We have $\sigma=-1+i \sqrt{\mu^{2}-1}$ in the notation of [17]. We note the equivalence of the representations under the replacement $h_{+} \rightarrow h_{-}$and $2 h_{+}-2=-2 h_{-}$. For the principal series, the inner product is simply the usual integral over the 2-sphere in Fubini-Study coordinates which matches the Klein-Gordon norm up to a constant factor. For $0<\mu^{2}<1$ we have the complementary series representations and the above results extend straightforwardly to that case.

\section{$7 \quad$ Relation to previous work}

Here we show the mode functions computed in ref. [10] form a non-unitary highest-weight representation, and therefore do not produce a unitary principal series representation of $\mathrm{SO}(3,1)$. To do so we first show that the generators of the special conformal transformations annihilate a mode function corresponding to the highest weight of the representation. The mode functions (eq. 2.19 of [10]) are parameterized by the tuple $(\Delta, \vec{w})$ where $\Delta$ is in general a complex number and $\vec{w}=\left(w_{x}, w_{y}\right) \in \mathbb{R}^{2}$. These mode functions are

$$
\phi_{\Delta}^{ \pm}\left(X^{\mu} ; \vec{w}\right)=\frac{4 \pi}{(i m)} \frac{\left(\sqrt{-X^{2}}\right)^{\Delta-1}}{(-q(\vec{w}) \cdot X \mp i \epsilon)^{\Delta}} K_{\Delta-1}\left(m \sqrt{X^{2}}\right) .
$$


Here $X^{\mu}$ are the usual flat coordinates of the $4 \mathrm{~d}$ Minkowski spacetime $\mathrm{M}_{4}$. The $q^{\mu}(\vec{w})$ is the following 4 -vector

$$
q^{\mu}(\vec{w})=\left(1+|\vec{w}|^{2}, 2 \vec{w}, 1-|\vec{w}|^{2}\right) .
$$

The conformal group $\mathrm{SO}(3,1)$ acts on the space of scalar functions defined on $\mathrm{M}_{4} \times \mathbb{R}^{2}$ by acting on $\mathrm{M}_{4}$ with the usual Lorentz transformation and on $\mathbb{R}^{2}$ with the $2 \mathrm{D}$ conformal transformations (2D translations, 2D rotations, dilatations and special conformal transformations):

$$
\phi_{\Delta}\left(X^{\mu} ; \vec{w}\right) \rightarrow \phi_{\Delta}\left(\Lambda_{\nu}^{\mu} X^{\nu} ; \vec{w}^{\prime}(\vec{w})\right) .
$$

Here $\Lambda_{\nu}^{\mu}$ is the Lorentz transformation corresponding to the $\mathrm{SO}(3,1)$ group element, and the $\vec{w}^{\prime}(\vec{w})$ is the conformal transformation corresponding to the $\operatorname{SO}(3,1)$ element. For special conformal transformation, it is

$$
\vec{w}^{\prime}=\frac{\vec{w}+|\vec{w}|^{2} \vec{b}}{1+2 \vec{b} \cdot \vec{w}+|\vec{b}|^{2}|\vec{w}|^{2}} .
$$

These are labeled by a vector $\vec{b} \in \mathbb{R}^{2}$.

Consider an infinitesimal group element near the identity

$$
\Lambda_{\nu}^{\mu}=\delta_{\nu}^{\mu}+(\delta \Lambda)^{\mu}{ }_{\nu}
$$

this has the corresponding infinitesimal transformation on $\mathbb{R}^{2}$

$$
\vec{w}^{\prime}=\vec{w}+\delta \vec{w} .
$$

In particular, for the special conformal transformation parameterized by an infinitesimal $\vec{b}$ we have the infinitesimal transformation

$$
\vec{w}^{\prime}=\vec{w}-2(\vec{w} \cdot \vec{b}) \vec{w}+|\vec{w}|^{2} \vec{b} .
$$

Following the conventions of [10] this corresponds to the infinitesimal Lorentz transformation where

$$
(\delta \omega)^{0}{ }_{i}=(\delta \omega)^{i}{ }_{0}=(\delta \omega)^{3}{ }_{i}=-(\delta \omega)^{i}{ }_{3}=b_{i}
$$

with all other components being zero. Here $i=x, y$ labels the indices of $\mathbb{R}^{2}$. In other words we have

$$
\left[\begin{array}{c}
X^{\prime 0} \\
X^{\prime i} \\
X^{\prime 3}
\end{array}\right]=\left[\begin{array}{c}
X^{0} \\
X^{i} \\
X^{3}
\end{array}\right]+\left[\begin{array}{c}
b_{i} X^{i} \\
b^{i}\left(X^{0}-X^{3}\right) \\
b_{i} X^{i}
\end{array}\right] .
$$

Taylor-expanding $\phi_{\Delta}\left(\Lambda_{\nu}^{\mu} X^{\nu} ; \vec{w}^{\prime}(\vec{w})\right)$ and substituting the expressions for $\delta \omega$ and $\delta \vec{w}$ above, we have

$$
\begin{aligned}
\phi_{\Delta}\left(\Lambda_{\nu}^{\mu} X^{\nu} ; \vec{w}^{\prime}(\vec{w})\right)= & \phi_{\Delta}\left(X^{\mu}+(\delta \omega)^{\mu}{ }_{\nu} X^{\nu} ; \vec{w}+\delta \vec{w}\right) \\
= & \phi_{\Delta}\left(X^{\nu} ; \vec{w}\right)+(\delta \omega)^{\mu}{ }_{\nu} X^{\nu}\left(\frac{\partial}{\partial X^{\mu}} \phi_{\Delta}\right)+\delta \vec{w} \cdot\left(\frac{\partial}{\partial \vec{w}} \phi_{\Delta}\right) \\
= & \phi_{\Delta}\left(X^{\nu} ; \vec{w}\right)+b_{i} X^{i}\left(\frac{\partial}{\partial X^{0}}+\frac{\partial}{\partial X^{3}}\right) \phi_{\Delta}+b^{i}\left(X^{0}-X^{3}\right)\left(\frac{\partial}{\partial X^{i}} \phi_{\Delta}\right) \\
& +\left(-2(\vec{w} \cdot \vec{b}) \vec{w}+|\vec{w}|^{2} \vec{b}\right) \cdot\left(\frac{\partial}{\partial \vec{w}} \phi_{\Delta}\right) .
\end{aligned}
$$


For infinitesimal $\vec{b}$ this evaluates to

$$
\delta \phi_{\Delta}=\frac{8 \pi \Delta}{i m} \vec{b} \cdot \vec{w} \frac{\left(\sqrt{-X^{2}}\right)^{\Delta-1}}{(-q(\vec{w}) \cdot X \mp i \epsilon)^{\Delta}} K_{\Delta-1}\left(m \sqrt{X^{2}}\right) .
$$

In particular, the special conformal transformations annihilate the mode functions when the weight $\vec{w}=(0,0)$. This implies [18] that the mode functions (7.1) form a highestweight representation of $\mathrm{SO}(3,1)$, and since $\mathrm{SO}(3,1)$ has only non-unitary highest-weight representations, these mode functions cannot form a unitary principal series.

\section{Discussion}

The results of the previous sections lead to a proposal for a holographic mapping between the 3D de Sitter modes and conformal operators on a two-sphere. Likewise, the construction may be lifted to 4D Minkowski spacetime.

\subsection{Holographic mapping between 3D de Sitter spacetime and a Euclidean 2-sphere}

For the case of a flat slicing, it was possible to define a bulk-to-boundary map and its inverse map [19] via a construction reminiscent of the LSZ reduction formula in asymptotically flat spacetime [20]. That construction does not extend immediately to the case of the sphere slicing, but we will see the Klein-Gordon inner product defined above can still be used to extract a natural set of operators living on the 2 -sphere.

We work with a scalar bulk field of mass $\mu$

$$
\phi(t, z, \bar{z})=\sum_{l=0}^{\infty} \sum_{m=-l}^{l} a_{l m} \phi_{l m}(t, z, \bar{z})+a_{l m}^{\dagger} \phi_{l m}^{\dagger}(t, z, \bar{z})
$$

where $\phi_{l m}$ is defined using the Euclidean vacuum modes (A.1) and $a_{l m}$ and $a_{l m}^{\dagger}$ are standard creation/annihilation operators. Let us consider a late-time sphere at $t=T$ and build the following inner product using the Klein-Gordon inner product in 3d de Sitter spacetime

$$
\mathcal{O}_{l m}=\left\langle\phi_{l m}(t), \phi(t, z, \bar{z})\right\rangle_{t=T}
$$

which identifies $\mathcal{O}_{l m}=a_{l m}$. Likewise one can define $\mathcal{O}_{l m}^{\dagger}=a_{l m}^{\dagger}$. One may view this mapping as a holographic map, with $l, m$ being dual variables to the coordinates on the 2 -sphere $z, \bar{z}$. The formula (8.1) can then be viewed as the inverse mapping reconstructing the bulk field in terms of boundary operators. The boundary operators will obey their usual commutation relations, and at the same time will transform as a representation of the unitary principal series as described above. All the above is established at the level of free field theory. Once interactions are included it seems difficult to view the resulting boundary theory as any kind of conventional field theory [21]. 


\subsection{Holographic mapping between celestial sphere and 4D Minkowski space- time}

This procedure can be extended to the $4 \mathrm{D}$ Minkowski case. However we will now have a continuous spectrum of allowed conformal weights $\Delta$ corresponding to the continuous spectrum of radial quantum numbers $p$. For a scalar field we can use the orthogonality of the radial mode functions (5.5) to project onto a particular $p$ eigenvalue and then follow the procedure of the previous subsection to build a boundary operator. The mode expansion of the bulk field is now

$$
\Phi(\eta, \rho, z, \bar{z})=\sum_{l=0}^{\infty} \sum_{m=-l}^{l} \int_{1}^{\infty} d p\left(a_{p l m} \Phi_{p l m}(\eta, \rho, z, \bar{z})+a_{p l m}^{\dagger} \Phi_{p l m}^{\dagger}(\eta, \rho, z, \bar{z})\right)
$$

where $a_{p l m}$ and $a_{p l m}^{\dagger}$ are annihilation and creation operators. Again we can define an operator on the celestial 2-sphere by constructing

$$
\mathcal{O}_{\Delta_{p}, l m}=\left\langle\Phi_{p l m}(\eta, \rho, z, \bar{z}), \Phi(\eta, \rho, z, \bar{z})\right\rangle=a_{p l m}
$$

where the operator transforms as a unitary principal series representation parameterized by $\Delta_{p}$. Likewise one may define a conjugate operator $\mathcal{O}_{\Delta_{p}, l m}^{\dagger}=a_{p l m}^{\dagger}$. We can therefore interpret this as a holographic map between the bulk 4D Minkowski spacetime and the boundary 2D celestial sphere. One ends up with a continuous family of boundary operators labelled by the radial quantum number $p$. As above, it is not clear how the construction extends to interacting theories.

\section{A Euclidean vacuum}

The closed form expression of the positive-frequency Euclidean modes has been computed in ref. [22], eq. 3.37, which we reproduce below in the interest of being self-contained. Translating the notations of ref. [22] to our notations, the time-dependent component of the (unnormalized) Euclidean modes of the 3d de Sitter spacetime is

$$
\phi_{l}^{E}(t)=\left(\cosh ^{l} t\right) e^{\left(l+1+i \sqrt{\mu^{2}-1}\right) t}{ }_{2} \mathrm{~F}_{1}\left(l+1, l+1+i \sqrt{\mu^{2}-1} ; 2 l+2 ; 1+e^{2 t}\right) .
$$

The identities [23] and [24] may be used provided we continue $t$ to the complex plane. It is then straightforward to verify that the linear combination of modes in (3.1)

$$
\phi_{l}^{E}(t) \propto \frac{i \pi}{2} \phi_{l, 1}(t)+\phi_{l, 2}(t)
$$

are the (unnormalized) positive-frequency modes corresponding to the Euclidean vacuum of the $3 \mathrm{~d}$ de Sitter spacetime upon continuing $t$ to real values. These when uplifted to the $4 \mathrm{~d}$ Minkowski spacetime will correspond to the Minkowski vacuum, since both are distinguished by the fact that their Wightman function has a Hadamard singularity [25]. The normalization factor is determined in section 5 . 
Open Access. This article is distributed under the terms of the Creative Commons Attribution License (CC-BY 4.0), which permits any use, distribution and reproduction in any medium, provided the original author(s) and source are credited.

\section{References}

[1] J. de Boer and S.N. Solodukhin, A holographic reduction of Minkowski space-time, Nucl. Phys. B 665 (2003) 545 [hep-th/0303006] [INSPIRE].

[2] D. Kapec, V. Lysov, S. Pasterski and A. Strominger, Semiclassical Virasoro symmetry of the quantum gravity $\mathcal{S}$-matrix, JHEP 08 (2014) 058 [arXiv: 1406.3312] [INSPIRE].

[3] D. Kapec, P. Mitra, A.-M. Raclariu and A. Strominger, $2 D$ stress tensor for $4 D$ gravity, Phys. Rev. Lett. 119 (2017) 121601 [arXiv: 1609.00282] [INSPIRE].

[4] C. Cheung, A. de la Fuente and R. Sundrum, $4 D$ scattering amplitudes and asymptotic symmetries from $2 D$ CFT, JHEP 01 (2017) 112 [arXiv:1609.00732] [INSPIRE].

[5] A. Strominger, The dS/CFT correspondence, JHEP 10 (2001) 034 [hep-th/0106113] [INSPIRE].

[6] A. Guijosa and D.A. Lowe, A new twist on dS/CFT, Phys. Rev. D 69 (2004) 106008 [hep-th/0312282] [INSPIRE].

[7] A. Guijosa, D.A. Lowe and J. Murugan, A prototype for dS/CFT, Phys. Rev. D 72 (2005) 046001 [hep-th/0505145] [INSPIRE].

[8] D.A. Lowe, q-deformed de Sitter/conformal field theory correspondence, Phys. Rev. D 70 (2004) 104002 [hep-th/0407188] [INSPIRE].

[9] S. Pasterski, S.-H. Shao and A. Strominger, Flat space amplitudes and conformal symmetry of the celestial sphere, Phys. Rev. D 96 (2017) 065026 [arXiv:1701.00049] [InSPIRE].

[10] S. Pasterski and S.-H. Shao, Conformal basis for flat space amplitudes, Phys. Rev. D 96 (2017) 065022 [arXiv:1705.01027] [INSPIRE].

[11] S. Pasterski, S.-H. Shao and A. Strominger, Gluon amplitudes as $2 d$ conformal correlators, Phys. Rev. D 96 (2017) 085006 [arXiv:1706.03917] [InSPIRE].

[12] W. Kelvin and P. Tait, Treatise on natural philosophy, Clarendon Press series, volume 1, Clarendon Press, Oxford, U.K. (1867).

[13] B. Allen, Vacuum states in de Sitter space, Phys. Rev. D 32 (1985) 3136 [inSPIRE].

[14] K. Goldstein and D.A. Lowe, A note on alpha vacua and interacting field theory in de Sitter space, Nucl. Phys. B 669 (2003) 325 [hep-th/0302050] [INSPIRE].

[15] K. Goldstein and D.A. Lowe, Real time perturbation theory in de Sitter space, Phys. Rev. D 69 (2004) 023507 [hep-th/0308135] [INSPIRE].

[16] N. Birrell, N. Birrell and P. Davies, Quantum fields in curved space, Cambridge Monographs on Mathematical Physics, Cambridge University Press, Cambridge, U.K. (1984).

[17] N.J. Vilenkin and A.U. Klimyk, Representation of Lie groups and special functions. Volume 2: class I representations, special functions and integral transforms, Springer, Dordrecht, The Netherlands (1993). 
[18] D. Simmons-Duffin, The conformal bootstrap, in Theoretical Advanced Study Institute in Elementary Particle Physics: new frontiers in fields and strings, World Scientific, Singapore (2016) [arXiv:1602.07982] [INSPIRE].

[19] A. Chatterjee and D.A. Lowe, Holographic operator mapping in dS/CFT and cluster decomposition, Phys. Rev. D 92 (2015) 084038 [arXiv:1503.07482] [InSPIRE].

[20] H. Lehmann, K. Symanzik and W. Zimmermann, On the formulation of quantized field theories, Nuovo Cim. 1 (1955) 205 [INSPIRE].

[21] A. Chatterjee and D.A. Lowe, dS/CFT and the operator product expansion, Phys. Rev. D 96 (2017) 066031 [arXiv:1612.07785] [INSPIRE].

[22] R. Bousso, A. Maloney and A. Strominger, Conformal vacua and entropy in de Sitter space, Phys. Rev. D 65 (2002) 104039 [hep-th/0112218] [INSPIRE].

[23] Associated Legendre of the first kind identity Mathematica function webpage, http://functions.wolfram.com/07.08.26.0007.01.

[24] Associated Legendre of the second kind identity Mathematica function webpage, http://functions.wolfram.com/07.11.26.0006.01.

[25] S.A. Fulling, M. Sweeny and R.M. Wald, Singularity structure of the two point function in quantum field theory in curved space-time, Commun. Math. Phys. 63 (1978) 257 [INSPIRE]. 